DIW BERLIN

Discussion

Papers
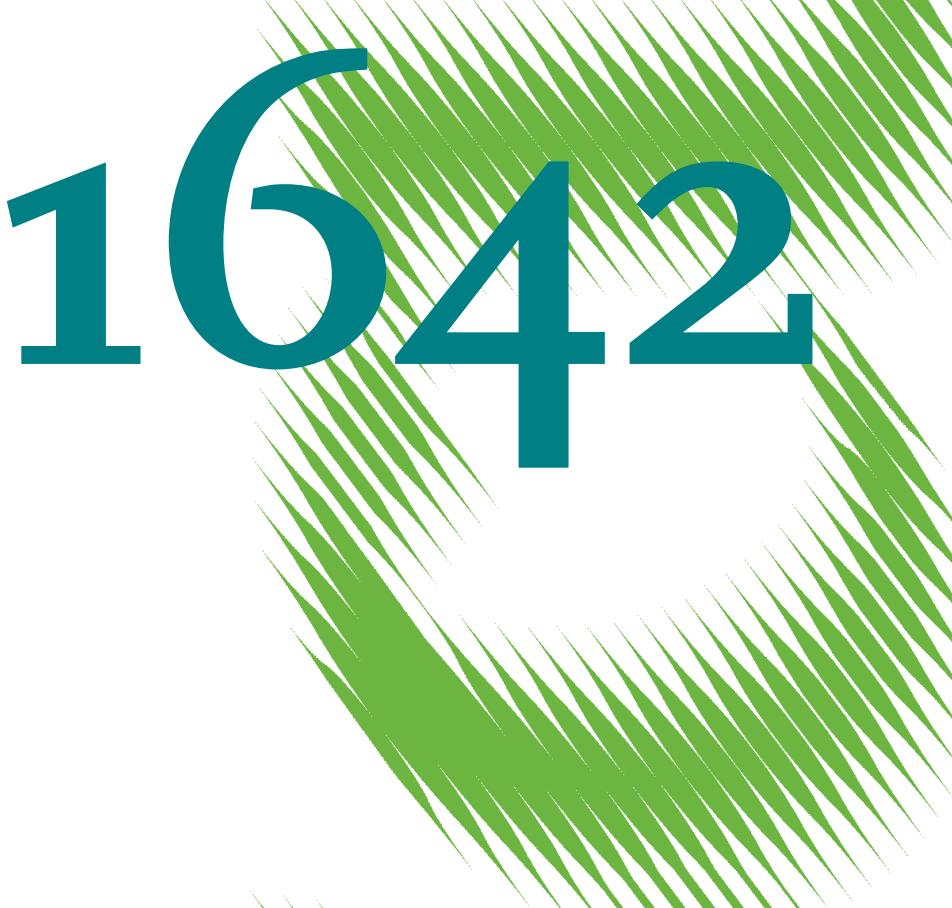

$M M$

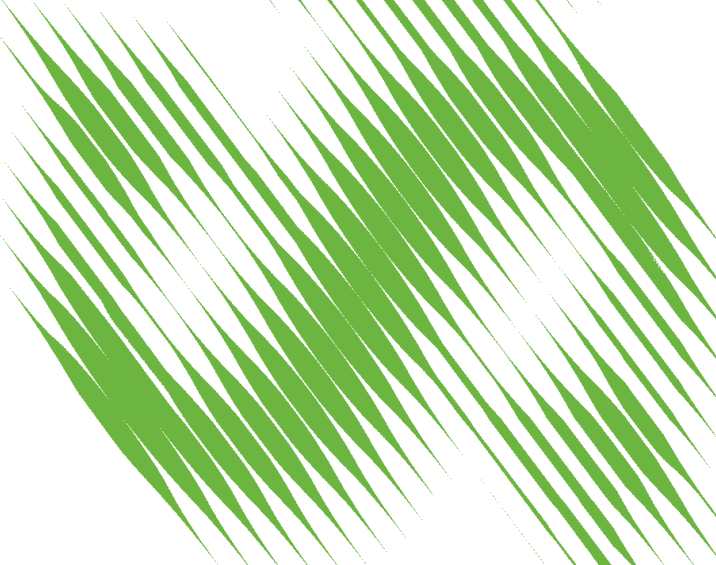

Estimation of Structural Impulse Responses: Short-Run versus Long-Run Identifying Restrictions 
Opinions expressed in this paper are those of the author(s) and do not necessarily reflect views of the institute.

IMPRESSUM

(C) DIW Berlin, 2017

DIW Berlin

German Institute for Economic Research

Mohrenstr. 58

10117 Berlin

Tel. +49 (30) $89789-0$

Fax +49 (30) $89789-200$

http://www.diw.de

ISSN electronic edition 1619-4535

Papers can be downloaded free of charge from the DIW Berlin website:

http://www.diw.de/discussionpapers

Discussion Papers of DIW Berlin are indexed in RePEc and SSRN:

http://ideas.repec.org/s/diw/diwwpp.html

http://www.ssrn.com/link/DIW-Berlin-German-Inst-Econ-Res.html 


\title{
Estimation of Structural Impulse Responses: Short-Run versus Long-Run Identifying Restrictions
}

\author{
Helmut Lütkepohl \\ DIW Berlin and Freie Universität Berlin \\ Mohrenstr. 58 \\ 10177 Berlin, Germany \\ email: hluetkepohl@diw.de \\ Anna Staszewska-Bystrova \\ University of Lodz \\ Rewolucji 1905r. 41 \\ 90-214 Lodz, Poland \\ email: emfans@uni.lodz.pl \\ Peter Winker \\ University of Giessen \\ Licher Str. 64 \\ 35394 Giessen, Germany \\ email: Peter.Winker@wirtschaft.uni-giessen.de
}

February 9, 2017

\begin{abstract}
There is evidence that estimates of long-run impulse responses of structural vector autoregressive (VAR) models based on long-run identifying restrictions may not be very accurate. This finding suggests that using short-run identifying restrictions may be preferable. We compare structural VAR impulse response estimates based on long-run and short-run identifying restrictions and find that long-run identifying restrictions can result in much more precise estimates for the structural impulse responses than restrictions on the impact effects of the shocks.
\end{abstract}

Key Words: Impulse responses, structural vector autoregressive model, longrun multipliers, short-run multipliers 


\section{Introduction}

Some authors have pointed out that standard estimators of the long-run multiplier matrix of a structural vector autoregressive (VAR) model identified with long-run restrictions may not be very precise (e.g., Faust and Leeper (1997), Christiano, Eichenbaum and Vigfusson (2006)). Moreover, it was found that confidence intervals around impulse responses from structural VARs identified by restrictions on the long-run effects of the structural shocks tend to be wide (e.g., Erceg, Guerrieri and Gust (2005), Gust and Vigfusson (2009)). Such results suggest that it may be preferable to use short-run restrictions for identifying shocks in structural VAR models, as argued by Faust and Leeper (1997). In practice, it may not be an option to replace long-run restrictions by short-run restrictions because the former are typically used when no convincing restrictions for the impact effects of the shocks are available. Hence, the question arises how much estimation precision is lost by not committing to identifying short-run restrictions and using long-run restrictions instead.

The aim of this study is to compare the relative estimation precision implied by short-run and long-run identifying restrictions for structural VAR models. Usually short-run and long-run restrictions will result in quite different structural impulse responses. In that case, comparing the relative accuracy is difficult. Therefore, we set up the following experiment to perform a fair comparison between the two alternative identification methods. We consider data generation processes (DGPs) and identifying restrictions which lead to exactly the same structural impulse responses. Then we have a proper basis for comparing the two alternative approaches. We use a Monte Carlo simulation experiment to compare the small sample mean squared errors (MSEs) of estimated impulse responses.

In practice the VAR order is unknown and may be infinite. Such features of a VAR model were seen as a main problem in some of the related literature that has pointed out problems with long-run identifying restrictions (e.g., Christiano et al. (2006)). Therefore we use model selection criteria for specifying the VAR order and we also consider infinite order VAR DGPs such that the finite order VAR model used for impulse response analysis is just an approximation to the true DGP.

We focus on simple recursive restrictions on the impact effects of the structural shocks and compare them to recursive restrictions on the long-run multipliers. Moreover, we consider bivariate and three-dimensional DGPs only. Thus, our DGPs have features of many models that have been used in structural VAR analysis in that they include a small number of variables and use recursive restrictions which are perhaps the most commonly used identifying restrictions in structural VAR analysis. We find that the relative accuracy of the impulse response estimates obtained with short-run and longrun identifying restrictions depends on specific characteristics of the DGP. As suggested in the aforementioned literature, short-run restrictions lead to more accurate estimates of the impact effects of shocks. However, long-run multipliers and impulse responses for intermediate and larger propagation horizons 
may be estimated more accurately when long-run identifying restrictions are used. The latter result holds specifically for persistent processes with a root close to one.

The study is structured as follows. Section 2 presents the basic model setup and the impulse responses of interest. The experimental design of the Monte Carlo study is considered in Section 3 and the results are discussed in Section 4. Section 5 concludes.

\section{The Model}

We consider the reduced-form VAR model of possibly infinite order,

$$
y_{t}=\nu+\sum_{i=1}^{\infty} A_{i} y_{t-i}+u_{t}
$$

where $y_{t}=\left(y_{1 t}, \ldots, y_{K t}\right)^{\prime}$, the $A_{i}, i=1,2, \ldots$, are $K \times K$ coefficient matrices, $\nu$ is a fixed $K \times 1$ intercept term and $u_{t}=\left(u_{1 t}, \ldots, u_{K t}\right)^{\prime}$ is a zero mean white noise error process such that $u_{t} \sim\left(0, \Sigma_{u}\right)$. The covariance matrix $\Sigma_{u}$ is positive definite.

For simplicity we exclude processes with integrated or cointegrated variables and assume that the VAR is stable and stationary satisfying

$$
\operatorname{det} A(z)=\operatorname{det}\left(I_{K}-\sum_{i=1}^{\infty} A_{i} z^{i}\right) \neq 0 \text { for } z \in \mathbb{C},|z| \leq 1
$$

such that the roots are bounded away from the unit circle and the process has the moving average (MA) representation

$$
y_{t}=A(1)^{-1} \nu+A(L)^{-1} u_{t}=\mu+\sum_{i=0}^{\infty} \Phi_{i} u_{t-i}
$$

where $\mu=A(1)^{-1} \nu, \Phi_{0}=I_{K}$ and $\sum_{i=0}^{\infty} \Phi_{i} L^{i}=A(L)^{-1}$. Clearly, the MA coefficient matrices are functions of the $A_{i}$ parameter matrices (e.g., Lütkepohl (2005)).

The structural shocks $\varepsilon_{t}$ are obtained by a linear transformation of the reduced form residuals $u_{t}$, that is, $\varepsilon_{t}=B^{-1} u_{t}$. The structural shocks are instantaneously uncorrelated and the variances are normalized to one. In other words, $\varepsilon_{t} \sim\left(0, I_{K}\right)$ and the matrix $B$ has to be such that $B B^{\prime}=$ $\Sigma_{u}$. The corresponding structural impulse responses are the elements of the coefficient matrices of the infinite order polynomial $A(L)^{-1} B=\sum_{i=0}^{\infty} \Theta_{i} L^{i}$. In our comparison of estimated impulse responses we focus on cumulated impulse responses $\Xi_{n}=\sum_{i=0}^{n} \Theta_{i}$ because these quantities are typically of interest when long-run restrictions are imposed. Note that the elements of $B=\Xi_{0}=\Theta_{0}$ are the impact multipliers or short-run multipliers of the structural shocks. We denote the matrix of long-run multipliers or total impact multipliers of the structural shocks by $\Xi_{\infty}=\sum_{i=0}^{\infty} \Theta_{i}=A(1)^{-1} B$. 
The relation $B B^{\prime}=\Sigma_{u}$ does not uniquely specify the transformation matrix $B$. For uniquely identifying these multipliers and, hence, the shocks, zero restrictions are imposed on $B$ in many structural VAR models. We consider recursive short-run restrictions obtained by choosing a lower-triangular matrix $B^{s}$ by a Cholesky decomposition of $\Sigma_{u}$. In other words,

$$
B^{s}=\operatorname{chol}\left(\Sigma_{u}\right),
$$

where chol denotes the Cholesky decomposition. These restrictions are the most common short-run identifying restrictions for structural shocks in VAR analysis.

Alternatively, we consider long-run restrictions imposed on the total impact multiplier matrix of impulse responses, $\Xi_{\infty}=A(1)^{-1} B$. Cumulated impulse responses are typically of interest when $y_{t}$ contains growth rates of economic variables. Imposing a zero restriction on the cumulated long-run effect of a shock on a growth rate implies that the underlying variable in the long-run will return to its initial value where it has come from before the shock occurred. Such a restriction indirectly constrains the impact effects of the shocks and, hence, can be used for identifying the structural shocks. In the spirit of Blanchard and Quah (1989), we choose $B^{l}$ such that the long-run multiplier matrix $\Xi_{\infty}$ is lower-triangular. More precisely, $B^{l}$ is chosen such that

$$
B^{l}=A(1) \operatorname{chol}\left(A(1)^{-1} \Sigma_{u} A(1)^{-1 \prime}\right) .
$$

This choice uniquely identifies the structural shocks.

It can be shown that the impulse responses implied by the short-run and long-run restrictions incorporated in $B^{s}$ and $B^{l}$, respectively, are identical if $A(1)$ and, hence, also $A(1)^{-1}$ are lower triangular and $A(L)$ is such that $A(1)^{-1} B^{s}$ has positive diagonal elements. Note that the matrix $A(1)^{-1} \Sigma_{u} A(1)^{-1 \prime}$ can be expressed as:

$$
A(1)^{-1} \Sigma_{u} A(1)^{-1^{\prime}}=A(1)^{-1} B^{s} B^{s \prime} A(1)^{-1^{\prime}}=A(1)^{-1} B^{s}\left(A(1)^{-1} B^{s}\right)^{\prime} .
$$

If $A(1)$ is lower triangular, $A(1)^{-1} B^{s}$ is the product of two lower-triangular matrices and, hence, it is also lower triangular. If all its diagonal elements are positive, it is the Cholesky factor of $A(1)^{-1} \Sigma_{u} A(1)^{-1^{\prime}}$ because the Cholesky decomposition uniquely decomposes a positive definite matrix in lower-triangular Cholesky factors with positive diagonal elements. Thus, if $A(1)^{-1} B^{s}$ has positive diagonal elements, the long-run restrictions imply the same matrix $B$ as the short-run restrictions:

$$
B^{l}=A(1) \operatorname{chol}\left(A(1)^{-1} \Sigma_{u} A(1)^{-1 \prime}\right)=A(1) A(1)^{-1} B^{s}=B^{s} .
$$

In practice, finite order $\operatorname{VAR}(p)$ models are fitted to the data and the approximating estimated process is used for impulse response analysis. While the true impulse responses are identical, the estimated impulse response functions will differ, if the alternative identification approaches are used, because 
the estimator $\widehat{A}(1)$ is not lower triangular and, hence, $\widehat{B}^{l}=\widehat{A}(1) \operatorname{chol}\left(\widehat{A}(1)^{-1} \widehat{\Sigma}_{u} \widehat{A}(1)^{-1 \prime}\right)$ differs from $\widehat{B}^{s}=\operatorname{chol}\left(\widehat{\Sigma}_{u}\right)$ even if both are based on the reduced-form ordinary least squares (OLS) estimates of the VAR parameters. This is true even if the true DGP is a finite order $\operatorname{VAR}(p)$ process and the true order $p$ is used in estimating the parameters.

Since $\widehat{B}^{l}$ involves the inverse $\widehat{A}(L)^{-1}$, it may not be estimated accurately if the matrix $A(1)$ is near-singular. This problem is well-known in the scalar case, where an estimator $\widehat{\alpha}^{-1}$ tends to have a large variance if the true parameter value $\alpha$ is close to zero. In that case, even if $\widehat{\alpha}$ is a very accurate estimator of $\alpha, 1 / \widehat{\alpha}$ may be a very imprecise estimator of $1 / \alpha$. By analogy, the estimator $\widehat{B}^{l}$ of the impact multiplier matrix $B$ may not be very precise if some variables are very persistent and the process has roots close to 1 , in which case $A(1)$ is near-singular.

Note that the structural impulse responses are estimated as $\widehat{A}(L)^{-1} \widehat{B}$, where $\widehat{B}$ is either $\widehat{B}^{s}$ or $\widehat{B}^{l}$. Since the estimator $\widehat{A}(L)$ is based on reduced-form parameters only, it is unaffected by just-identifying structural restrictions. Thus, for the estimation precision of the structural impulse responses the crucial question is how accurately we are able to estimate the matrix $B$. Of course, it is possible that if the estimator of $B^{s}$ turns out to be more precise than that based on $B^{l}$, the latter estimator may still lead to more accurate estimates of impulse responses at propagation horizons greater than 0 . In the next section we explore the small sample accuracy of structural impulse responses.

\section{Monte Carlo Setup}

The aim of the Monte Carlo experiment is to compare the estimation precision for the two competing identification approaches. We use DGPs characterized by a lower-triangular matrix $A(1)$, for which the true impulse responses obtained using the short-run and long-run restrictions are the same. In the simulations, we study MSEs of the OLS estimators of the coefficients of $B^{s}, B^{l}, \Xi_{n}^{s}$ and $\Xi_{n}^{l}$, including $\Xi_{\infty}^{s}=A(1)^{-1} B^{s}, \Xi_{\infty}^{l}=A(1)^{-1} B^{l}$. We focus on those elements which are not restricted to zero for either type of identification scheme and present the results as relative MSEs,

$$
\frac{\operatorname{MSE}\left(\widehat{B}_{i j}^{s}\right)}{\operatorname{MSE}\left(\widehat{B}_{i j}^{l}\right)} \text { and } \frac{\operatorname{MSE}\left(\widehat{\Xi}_{n, i j}^{s}\right)}{\operatorname{MSE}\left(\widehat{\Xi}_{n, i j}^{l}\right)} \text {, }
$$

where a subscript $i j$ denotes the $i j^{\text {th }}$ element of the respective matrix and the superscripts $s$ and $l$ denote estimates based on short- and long-run restrictions, respectively.

In the experiments, the parameters of finite-order $\operatorname{VAR}(p)$ models with intercepts are estimated. The estimator for $\Sigma_{u}$ is $\widehat{\Sigma}_{u}=\frac{1}{T-K p-1} \widehat{U} \widehat{U}^{\prime}$, where $\widehat{U}=\left(\widehat{u}_{1}, \ldots, \widehat{u}_{T}\right)$ is a $K \times T$ matrix of OLS residuals. We choose the lag order $p$ by Akaike's information criterion AIC using a maximum lag order of 
12 and report results for sample size $T=100$. The number of replications is fixed at 10,000. All DGPs are Gaussian.

The first type of the data generating process (DGP1) is:

$$
y_{t}=\left[\begin{array}{cc}
\alpha_{11} & 0 \\
0.5 & 0.5
\end{array}\right] y_{t-1}+u_{t}, \quad u_{t} \sim \text { i.i.d. } \mathcal{N}\left(0,\left[\begin{array}{cc}
1 & \sigma_{12} \\
\sigma_{12} & 1
\end{array}\right]\right)
$$

where $\alpha_{11} \in\{-0.9,-0.5,0,0.5,0.9\}$ and $\sigma_{12} \in\{0.3,0.7\}$. This type of DGP has been used in a number of other studies of estimation and inference properties of structural impulse responses (see Kilian (1998), Lütkepohl, Staszewska-Bystrova and Winker (2015a, 2015b)). The parameter $\alpha_{11}$ determines the persistence of the process. A value close to one implies a process with a root near unity and a near-singular matrix $A(1)$, while smaller values of $\alpha_{11}$ imply a well-conditioned $A(1)$ matrix. As mentioned earlier, identifying long-run restrictions are often used for variables in growth rates which are typically not very persistent. There may still be some persistence in variables whose long-run responses are not restricted. Therefore we consider a value of $\alpha_{11}$ of 0.9 . If some variables are very persistent and there are autoregressive unit roots, one would typically consider modelling and estimation techniques for cointegrated variables. In particular, one would consider other types of long-run restrictions for identifying the structural shocks. Hence, we do not consider values of $\alpha_{11}$ greater than 0.9 .

For DGP1 the matrix of impact effects of the structural shocks is

$$
B=\operatorname{chol}\left(\Sigma_{u}\right)=\left[\begin{array}{ll}
1 & 0 \\
\sigma_{12} & \sqrt{1-\sigma_{12}^{2}}
\end{array}\right]
$$

and the matrix of long-run multipliers is

$$
\Xi_{\infty}=A(1)^{-1} B=\left[\begin{array}{ll}
\frac{1}{1-\alpha_{11}} & 0 \\
\frac{1}{1-\alpha_{11}}+2 \sigma_{12} & 2 \sqrt{1-\sigma_{12}^{2}}
\end{array}\right] .
$$

For all parameter values used in the simulations the diagonal elements of $\Xi_{\infty}$ are positive such that $B^{l}=B^{s}$.

Our second DGP (DGP2) is three dimensional and has the form:

$$
y_{1}=\left[\begin{array}{ccc}
\alpha_{11} & 0 & 0 \\
0.3 & 0.7 & 0 \\
0.2 & 0.2 & 0.5
\end{array}\right] y_{t-1}+u_{t}, \quad u_{t} \sim \text { i.i.d. } \mathcal{N}\left(0,\left[\begin{array}{ccc}
1 & 0.4 & 0.3 \\
0.4 & 1 & 0 \\
0.3 & 0 & 1
\end{array}\right]\right)
$$

where $\alpha_{11} \in\{-0.9,-0.5,0,0.5,0.9\}$. The persistence of the process is determined by the diagonal elements of the VAR parameter matrix $A_{1}$. Since the second and third diagonal elements are fixed at values not very close to one, the persistence of the process varies with $\alpha_{11}$. A value of $\alpha_{11}$ close to one implies a potential invertibility problem for $A(1)$. The largest value of $\alpha_{11}=0.9$ is close to one but still far enough away to ensure a well-conditioned matrix $A(1)$. 
The impact multiplier matrix of DGP2 is

$$
B=\operatorname{chol}\left(\Sigma_{u}\right)=\left[\begin{array}{rrr}
1 & 0 & 0 \\
0.4 & 0.9165 & 0 \\
0.3 & -0.1309 & 0.9449
\end{array}\right]
$$

and the long-run multipliers are

$$
\Xi_{\infty}=A(1)^{-1} B=\left[\begin{array}{ccc}
\frac{1}{1-\alpha_{11}} & 0 & 0 \\
\frac{1}{1-\alpha_{11}}+1.3333 & 3.0551 & 0 \\
\frac{0.8}{1-\alpha_{11}}+1.1333 & 0.9602 & 1.8898
\end{array}\right] .
$$

Note that the diagonal elements of $\Xi_{\infty}$ are positive implying that the shortand long-run identifying restrictions lead to identical impulse responses.

The third DGP (DGP3) is the bivariate MA $(1)(\operatorname{VAR}(\infty))$ process

$$
y_{t}=u_{t}+\left[\begin{array}{cc}
\alpha_{11} & 0 \\
0.5 & 0.5
\end{array}\right] u_{t-1}, \quad u_{t} \sim \text { i.i.d. } \mathcal{N}\left(0,\left[\begin{array}{cc}
1 & \sigma_{12} \\
\sigma_{12} & 1
\end{array}\right]\right)
$$

where $\alpha_{11} \in\{-0.9,-0.5,0,0.5,0.9\}$ and $\sigma_{12} \in\{0.3,0.7\}$.

The matrix of impact effects of the structural shocks is the same as in the case of DGP1:

$$
B=\operatorname{chol}\left(\Sigma_{u}\right)=\left[\begin{array}{ll}
1 & 0 \\
\sigma_{12} & \sqrt{1-\sigma_{12}^{2}}
\end{array}\right]
$$

and the matrix of long-run multipliers is

$$
\Xi_{\infty}=A(1)^{-1} B=\left[\begin{array}{ll}
1+\alpha_{11} & 0 \\
0.5+1.5 \sigma_{12} & 1.5 \sqrt{1-\sigma_{12}^{2}}
\end{array}\right]
$$

Again, for all parameter values used in the simulations the diagonal elements of $\Xi_{\infty}$ are positive such that $B^{l}=B^{s}$.

\section{Monte Carlo Results}

The simulation results for the three DGPs are presented in Tables 1 - 3 for the impact and long-run multipliers and in Figures 1 - 3 for small and intermediate propagation horizons. As expected, the short-run multipliers are estimated more efficiently when zero restrictions are imposed on the impact multiplier matrix $B$ directly. All the MSE ratios for the elements of $B$ are smaller than one for all parameter values in Tables 1 - 3. It is remarkable that for DGP1 variations in $\alpha_{11}$ in the range from -0.9 to 0.9 do not affect the relative estimation precision of the short-run multipliers substantially if the reduced-form residual correlation is small (see the results for DGP1 and $\sigma_{12}=0.3$ in Table 1). Increasing the reduced-form residual correlation to $\sigma_{12}=0.7$ makes a difference, however. In that case, the estimation precision based on short-run restrictions improves for $B_{21}$ and $B_{22}$ when $\alpha_{11}$ moves closer to one. 
The results for DGP2 and DGP3 are similar in that all elements of $B$ are estimated more accurately if short-run restrictions are imposed for identification. All related MSE ratios in Tables 2 and 3 are clearly smaller than one. For some elements of $B$ the gains in precision are quite dramatic (see, e.g., the relative MSEs of $B_{21}$ in Table 2). Note that the reduced-form residual correlations of DGP2 are rather small. So the results for DGP2 are best compared to those of DGP1 with $\sigma_{12}=0.3$.

The picture changes for all three DGPs when we consider the estimated long-run multipliers. In Tables 1 - 3 the MSE ratios are partly clearly larger than 1, implying that long-run identifying restrictions lead to more accurate estimates of the long-run multipliers. In some cases the MSE reductions due to long-run restrictions are rather dramatic (see $\Xi_{\infty, 22}$ in Table 1 , $\Xi_{\infty, 22}$, $\Xi_{\infty, 32}$ and $\Xi_{\infty, 33}$ in Table 2 and $\Xi_{\infty, 22}$ in Table 3 ). For the long-run multipliers the relative estimation precision depends substantially on the persistence of the process which is governed by $\alpha_{11}$ for DGP1 and DGP2. In fact, the MSE ratios increase with $\alpha_{11}$ implying that using long-run restrictions can be beneficial for estimating the long-run multipliers of more persistent processes. Moreover, an $\alpha_{11}$ close to 1 for DGP3 implies that an accurate approximation requires a large VAR order. This feature apparently improves the value of long-run restrictions relative to short-run restrictions, as seen in Table 3 .

In summary, if one is interested in the long-run effects of structural shocks, using long-run restrictions can result in more precise estimates. The gains in estimation precision are especially large for persistent processes and they can also be substantial if the true DGP is an infinite order VAR process.

It is also interesting to look at the MSE ratios of impulse responses for short and intermediate propagation horizons in Figures 1 - 3. They show that the relative estimation precision often is close to the value for the long-run multipliers already after a few periods. Thus, even though the MSE ratios are smaller than 1 for the impact multipliers they are in many cases greater than 1 after a few propagation periods for those cases where the long-run multipliers have MSE ratios greater than 1 . It is remarkable that this result also holds for DGP3 which is an infinite-order VAR process and, hence, the empirical model only approximates the DGP (see Figure 3). This result is in contrst to the view that long-run identifying restrictions are particularly problematic for infinite-order VARs which are approximated by finite order models (see Christiano et al. (2006)).

We have done a range of further simulations which confirm our general results. First, we have also used larger samples of size $T=200$ and obtained similar, qualitatively identical results to those for $T=100$. Therefore we do not show them in tables. Second, we have done simulations using the true VAR order for DGP1 and DGP2. The results were numerically similar to those in Tables 1 and 2 respectively. In other words, for these DGPs it does not make much difference for the impulse response estimates whether the true VAR order is used or the order is estimated by AIC, if a sample of size $T=$ 100 is available. Third, structural impulse responses are often based on biasadjusted estimators of the VAR parameters because they were found to imply 
more precise estimates and confidence intervals for impulse responses (see, e.g., Kilian (1998)). We have also worked with bias-adjusted estimates in some of our simulations. They did not lead to MSE improvements, however. In fact, in most cases the MSEs were inferior to the corresponding OLS results, in some cases even substantially so. Therefore we do not present the results.

Despite our limited simulation design, we emphasize that our Monte Carlo results have brought about an insight that is not clear from the related literature, namely that long-run restrictions can lead to much more precise estimates of some impulse responses than short-run restrictions if comparable impulse responses are considered.

\section{Conclusions}

In this study we have compared the precision of structural impulse response estimates based on short-run and long-run identifying restrictions. In the structural VAR literature concerns have been raised regarding the estimation accuracy of impulse response estimates when long-run identifying restrictions are used, in particular for persistent processes. Since many macro variables are quite persistent, the use of long-run identifying restrictions has been questioned.

To see whether long-run restrictions are more problematic than short-run restrictions in estimating impulse responses, we have simulated processes for which short-run and long-run restrictions lead to identical impulse responses. We find that the impact multipliers are indeed estimated more accurately when identification is based on short-run restrictions. However, if intermediate or longer propagation horizons are of interest, this result is not maintained. We find that in many cases the impulse responses at intermediate propagation horizons and long-run multipliers are estimated much more precisely in terms of MSE than the corresponding quantities obtained with short-run restrictions. Particularly substantial MSE improvements from using long-run restrictions are obtained for more persistent processes.

\section{Acknowledgements}

The research for this paper was partly carried out while the first author was a Bundesbank Professor at the Freie Universität Berlin. Financial support was provided by the Deutsche Forschungsgemeinschaft through SFB 649 "Economic Risk" and the National Science Center, Poland (NCN) through HARMONIA 6: UMO-2014/14/M/HS4/00901.

\section{References}

Blanchard, O. and Quah, D. (1989). The dynamic effects of aggregate demand and supply disturbances, American Economic Review 79: 655- 
673.

Christiano, L. J., Eichenbaum, M. and Vigfusson, R. J. (2006). Alternative procedures for estimating vector autoregressions identified with long-run restrictions, Journal of the European Economic Association 4: 475-483.

Erceg, C., Guerrieri, L. and Gust, C. (2005). Can long-run restrictions identify technology shocks?, Journal of the European Economic Association 3: $1237-1278$.

Faust, J. and Leeper, E. (1997). When do long-run identifying restrictions give reliable results?, Journal of Business Economics and Statistics 15: $345-353$.

Gust, C. and Vigfusson, R. (2009). The power of long-run VARs. International Finance Discussion Paper No. 978.

Kilian, L. (1998). Small-sample confidence intervals for impulse response functions, Review of Economics and Statistics 80: 218-230.

Lütkepohl, H. (2005). New Introduction to Multiple Time Series Analysis, Springer-Verlag, Berlin.

Lütkepohl, H., Staszewska-Bystrova, A. and Winker, P. (2015a). Comparison of methods for constructing joint confidence bands for impulse response functions, International Journal for Forecasting 31: 782-798.

Lütkepohl, H., Staszewska-Bystrova, A. and Winker, P. (2015b). Confidence bands for impulse responses: Bonferroni versus Wald, Oxford Bulletin of Economics and Statistics 77: 800-821. 
Table 1: Relative MSEs for the OLS estimators of unrestricted elements of $B$ and $\Xi_{\infty}$ comparing the two identifications schemes for DGP1. The sample size is $T=100$, the number of Monte Carlo replications is 10,000, the lag order $p$ is estimated using AIC.

\begin{tabular}{rrrrrrrr}
\hline \hline & & \multicolumn{6}{c}{ Impulse responses } \\
\cline { 3 - 8 }$\sigma_{12}$ & $\alpha_{11}$ & $B_{11}$ & $B_{21}$ & $B_{22}$ & $\Xi_{\infty, 11}$ & $\Xi_{\infty, 21}$ & $\Xi_{\infty, 22}$ \\
\hline 0.3 & -0.9 & 0.7959 & 0.2873 & 0.6400 & 0.9668 & 0.4333 & 1.3397 \\
& -0.5 & 0.8073 & 0.2884 & 0.6485 & 0.9940 & 0.4804 & 1.3255 \\
& 0.0 & 0.8061 & 0.2906 & 0.6532 & 0.9970 & 0.5394 & 1.5743 \\
& 0.5 & 0.8039 & 0.2889 & 0.6395 & 1.0072 & 0.7060 & 2.3765 \\
& 0.9 & 0.8010 & 0.2884 & 0.6378 & 0.9877 & 0.9588 & 23.6527 \\
0.7 & -0.9 & 0.8515 & 0.4364 & 0.2832 & 0.9992 & 0.6310 & 2.3636 \\
& -0.5 & 0.8607 & 0.4321 & 0.2746 & 1.0063 & 0.6469 & 2.5350 \\
& 0.0 & 0.8389 & 0.4083 & 0.2545 & 1.0057 & 0.6866 & 3.1317 \\
& 0.5 & 0.8249 & 0.3790 & 0.2193 & 1.0069 & 0.8045 & 5.0748 \\
& 0.9 & 0.7794 & 0.3235 & 0.1774 & 0.9913 & 0.9744 & 45.8236 \\
\hline
\end{tabular}

Note: In the simulations, two Monte Carlo samples were substituted with new samples to avoid nonstationarity of the estimated system.
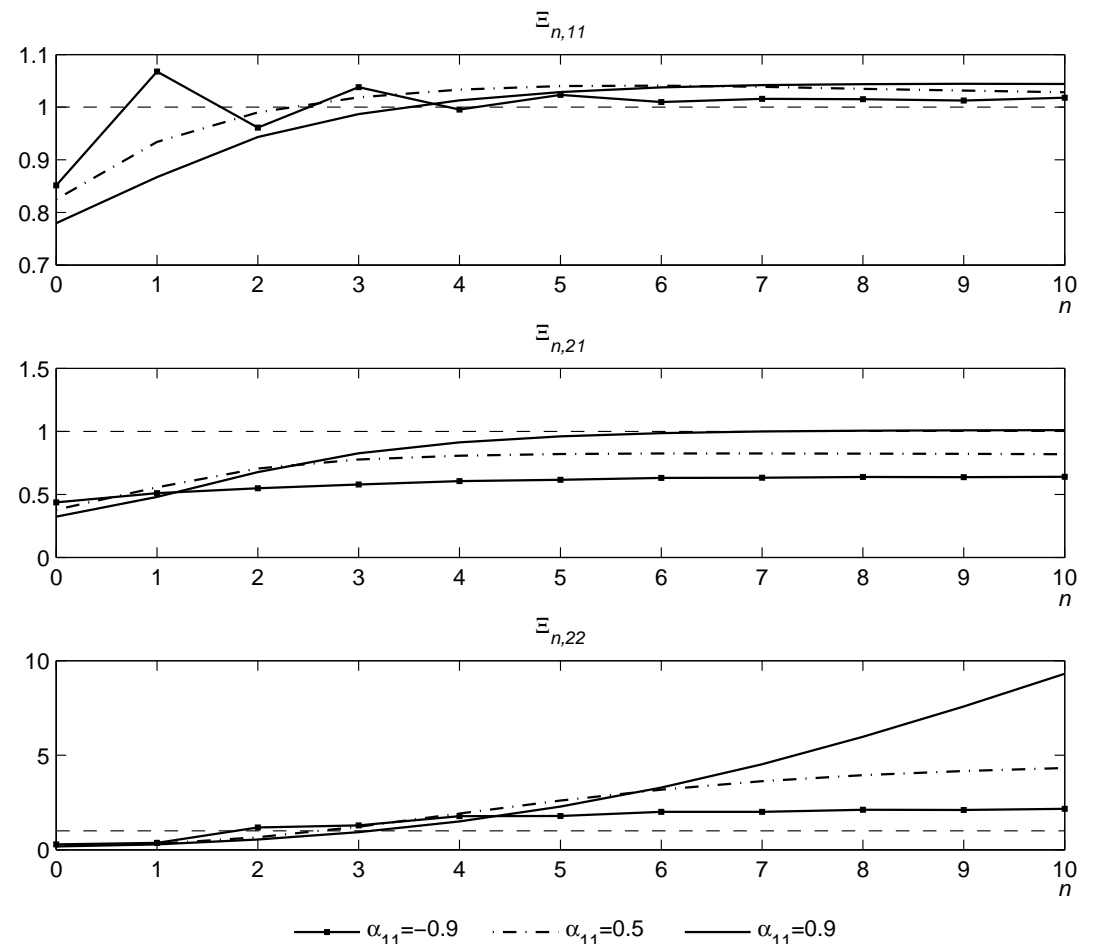

Figure 1: Relative MSEs for the OLS estimators of cumulated impulse responses for DGP1 with selected values of $\alpha_{11}, \sigma_{12}=0.7, T=100$ and lag order estimated using AIC. 
Table 2: Relative MSEs for the OLS estimators of unrestricted elements of $B$ and $\Xi_{\infty}$ comparing the two identifications schemes for DGP2. The sample size is $T=100$, the number of Monte Carlo replications is 10,000 , the lag order $p$ is estimated using AIC.

\begin{tabular}{rrrrrrr}
\hline \hline & \multicolumn{6}{c}{ Impulse responses } \\
\cline { 2 - 7 }$\alpha_{11}$ & $B_{11}$ & $B_{21}$ & $B_{22}$ & $B_{31}$ & $B_{32}$ & $B_{33}$ \\
\hline-0.9 & 0.6001 & 0.2022 & 0.4326 & 0.3128 & 0.2710 & 0.5831 \\
-0.5 & 0.6141 & 0.2040 & 0.4362 & 0.3106 & 0.2740 & 0.5853 \\
0.0 & 0.6079 & 0.2036 & 0.4237 & 0.3085 & 0.2734 & 0.5795 \\
0.5 & 0.5968 & 0.2029 & 0.4006 & 0.3081 & 0.2735 & 0.5648 \\
0.9 & 0.5979 & 0.2076 & 0.3710 & 0.3093 & 0.2720 & 0.5519 \\
& $\Xi_{\infty, 11}$ & $\Xi_{\infty, 21}$ & $\Xi_{\infty, 22}$ & $\Xi_{\infty, 31}$ & $\Xi_{\infty, 32}$ & $\Xi_{\infty, 33}$ \\
\cline { 2 - 7 }-0.9 & 1.0295 & 0.3965 & 1.3008 & 0.4626 & 1.0280 & 1.7103 \\
-0.5 & 1.0281 & 0.4189 & 1.3365 & 0.4873 & 1.0579 & 1.7792 \\
0.0 & 1.0352 & 0.4657 & 1.4492 & 0.5358 & 1.1833 & 1.9459 \\
0.5 & 1.0313 & 0.5875 & 1.9049 & 0.6571 & 1.6423 & 2.5997 \\
0.9 & 0.9481 & 0.9052 & 11.9891 & 0.9122 & 11.8429 & 19.4528 \\
\hline
\end{tabular}

Note: In the simulations, one Monte Carlo sample was substituted with a new one to avoid nonstationarity of the estimated system.
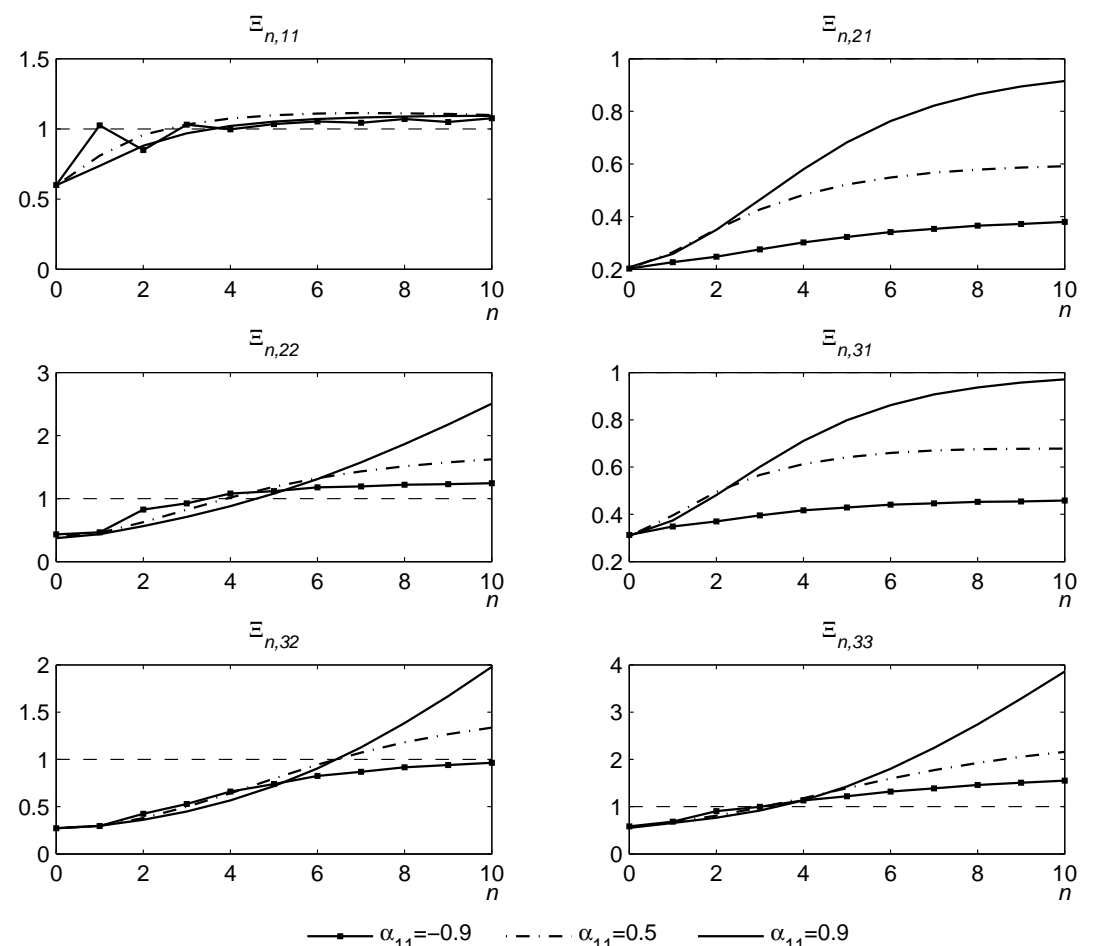

Figure 2: Relative MSEs for the OLS estimators of cumulated impulse responses for DGP2 with selected values of $\alpha_{11}, T=100$ and lag order estimated using AIC. 
Table 3: Relative MSEs for the OLS estimators of unrestricted elements of $B$ and $\Xi_{\infty}$ comparing the two identifications schemes for DGP3. The sample size is $T=100$, the number of Monte Carlo replications is 10,000 , the lag order $p$ is estimated using AIC.

\begin{tabular}{rrrrrrrr}
\hline \hline & & \multicolumn{7}{c}{ Impulse responses } \\
\cline { 5 - 9 }$\sigma_{12}$ & $\alpha_{11}$ & $B_{11}$ & $B_{21}$ & $B_{22}$ & $\Xi_{\infty, 11}$ & $\Xi_{\infty, 21}$ & $\Xi_{\infty, 22}$ \\
\hline 0.3 & -0.9 & 0.4540 & 0.0652 & 0.7053 & 0.7899 & 0.2092 & 0.8482 \\
& -0.5 & 0.7716 & 0.2139 & 0.6284 & 0.9099 & 0.5283 & 1.1265 \\
& 0.0 & 0.7643 & 0.2762 & 0.5893 & 0.9857 & 0.6710 & 1.3907 \\
& 0.5 & 0.6862 & 0.2233 & 0.4894 & 0.9932 & 0.7054 & 1.4434 \\
& 0.9 & 0.6143 & 0.2253 & 0.3444 & 0.9643 & 0.6710 & 1.5937 \\
0.7 & -0.9 & 0.1492 & 0.0323 & 0.0605 & 0.5322 & 0.2005 & 0.2832 \\
& -0.5 & 0.7742 & 0.2363 & 0.1549 & 0.9393 & 0.6322 & 1.3441 \\
& 0.0 & 0.7546 & 0.3250 & 0.1726 & 0.9809 & 0.7551 & 2.6451 \\
& 0.5 & 0.6702 & 0.2624 & 0.1278 & 0.9915 & 0.8009 & 2.8307 \\
& 0.9 & 0.6604 & 0.3031 & 0.0862 & 0.9621 & 0.8162 & 3.2410 \\
\hline
\end{tabular}
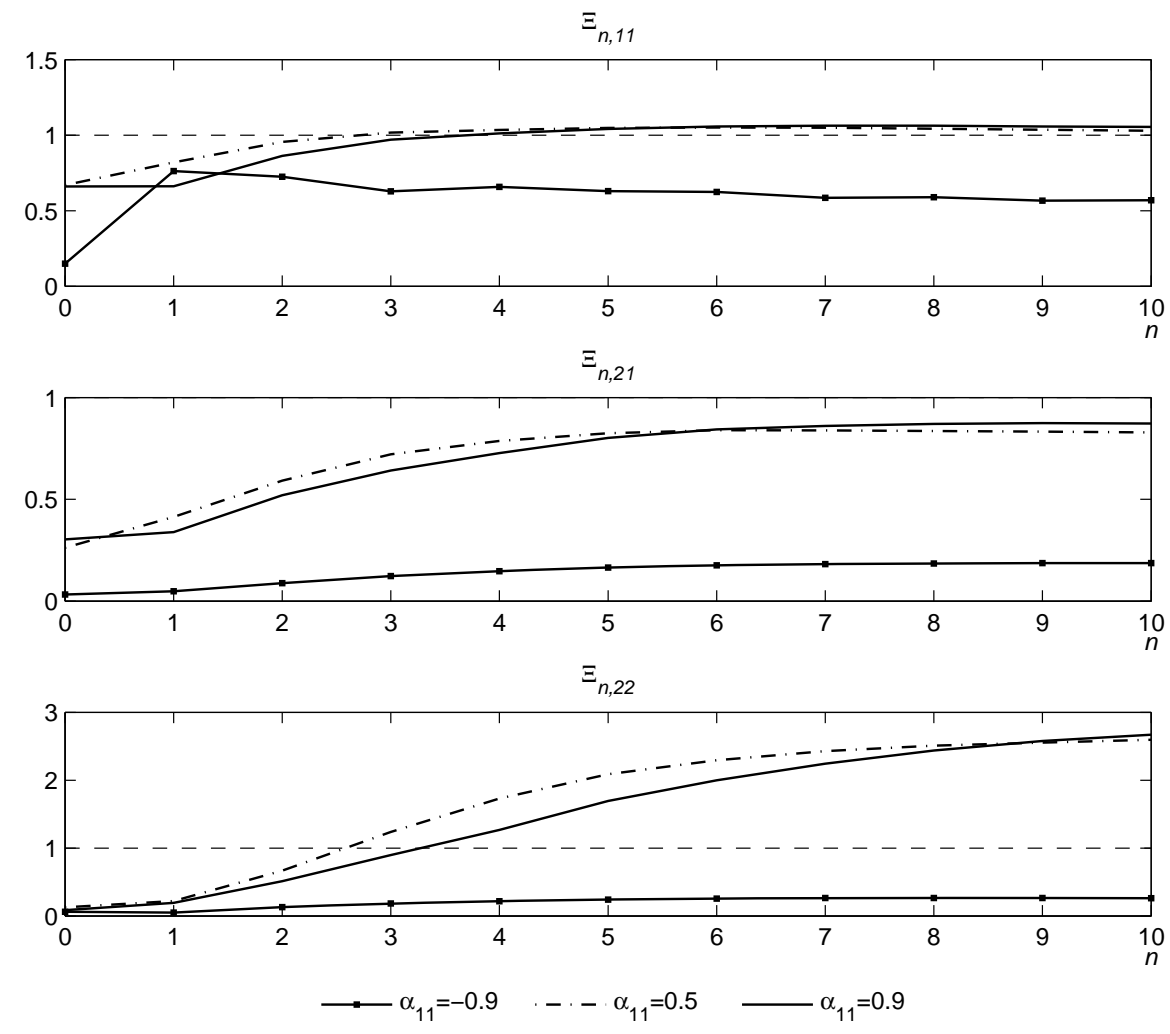

Figure 3: Relative MSEs for the OLS estimators of cumulated impulse responses for DGP3 with selected values of $\alpha_{11}, \sigma_{12}=0.7, T=100$ and lag order estimated using AIC. 